\title{
I mpacto de la Especialidad en Bioestadística sobre su claustro y sobre la Salud Pública Cubana, 1974-2006
}

\author{
I mpact of Biostatistics on the faculty in charge of this \\ specialty and on the Cuban public health care, 1974-2000
}

\author{
Lourdes Couturejuzón González '; Miday Columbié Pileta"
}

\author{
'Especialista de II Grado en Bioestadística. Profesora Auxiliar. Escuela Nacional de \\ Salud Pública. La Habana, Cuba. \\ "Especialista de I Grado en Bioestadística. Instructora. Escuela Nacional de Salud \\ Pública. La Habana, Cuba.
}

\section{RESUMEN}

Objetivo Evaluar el impacto de la Especialidad de Bioestadística sobre su claustro de profesores y sobre la Salud Pública Cubana.

Métodos Se realizó un estudio descriptivo y retrospectivo. Se construyeron dos modelos de entrevista personal para la obtención de la información: uno dirigido a los profesores integrantes del claustro de la especiallidad y el otro a expertos en el área de la salud pública. Se sometió a criterio de expertos la idoneidad de ambos modelos. El procesamiento de la información proveniente de las entrevistas realizadas se efectuó manualmente a través de un análisis de contenido.

Resultados Los profesores opinaron que el hecho de pertenecer al claustro de la especialidad constituye una de las fuentes más importantes en su formación y desarrollo que los obliga a mantener una elevada preparación científica, tecnológica y pedagógica, así como al perfeccionamiento de su desempeño como investigadores y docentes. Los expertos opinaron que el impacto de la Especialidad en la Salud Pública Cubana se aprecia en la calidad de la información estadística que se brinda en todos los niveles del Sistema Nacional de Salud, en el aumento de la calidad de las investigaciones y eventos científicos realizados en el sector de la salud, en la elevación de la calidad como investigadores y docentes de otros profesionales de la 
salud, en la creación del Comité Académico y del Grupo Nacional de la Especialidad, así como en la realización de eventos científicos propios de esta.

Conclusiones La Especialidad en Bioestadística tuvo impacto positivo sobre las esferas exploradas.

Palabras clave: Impacto educacional, evaluación educacional, evaluación de impacto, Especialidad de Bioestadística.

\section{ABSTRACT}

Objective To evaluate the impact of Biostatistics on the faculty in charge of this specialty and on the Cuban public health.

Methods A retrospective and descriptive study was conducted. Two personal interviews models were designed to gather information; one aimed at professors in this specialty and the other aimed at experts in the public health area. The suitability of both models was submitted to the expert criteria. Gathered information was manually processed on the basis of a contents analysis.

Results The professors stated that being part of the faculty in charge of this specialty is one of the most important sources for their formation and development since this forces them to keep a high level of scientific, technological and teaching preparation as well as the improvement of their performance as both researchers and educators. On the other hand, the experts pointed out that the impact of this specialty on the public health in Cuba is expressed in the quality of the statistical information provided at all levels of the national health care system, in the increased quality of research works carried out and scientific events held in this sector, in the improved performance of other health professionals as researchers and professors, in the creation of the Academic Commission and the National Group of this specialty and also in the holding of scientific events in this field.

Conclusions Biostatistics specialty had a positive impact on the explored spheres.

Key words: Educational impact, educational assessment, impact assessment, biostatistics specialty.

\section{NTRODUCCI ÓN}

La Estadística es una ciencia tan antigua como la escritura que nace con la necesidad del hombre de contar, realizar recuentos y censos.

Aunque sus orígenes se pierden en la antigüedad, no es hasta mediados del siglo XVII que la investigación y la práctica médica dieron lugar a un medio propicio para el desarrollo de esta disciplina.

En Cuba el desarrollo de las estadísticas médicas tiene alrededor de 4 siglos de evolución, comprende desde noticias aisladas en la época de la colonia y encontradas en las Actas Capitulares, hasta el estado actual de reconocimiento internacional en la calidad y confiabilidad de los datos ofrecidos. ${ }^{1}$ 
La Bioestadística es una de las ramas de la medicina que a pesar de tener antecedentes remotos, hace muy poco tiempo se consolidó como ciencia y se dio a conocer en el mundo. En la actualidad su aplicación constituye un recurso imprescindible para cualquier directivo sea o no del ámbito sanitario.

La especialidad surge en Cuba a partir de 1970, cuando se puso de manifiesto la necesidad de crear un profesional capaz de procesar, analizar e interpretar los datos estadísticos que ya eran emitidos por los técnicos a todo lo largo y ancho del país.

En 1971 comienza a formarse los primeros especialistas con un año común a otras especialidades de salud pública. En 1974 se oficializa como especialidad independiente y se elabora un plan de estudio basado en las experiencias de algunos conocedores del tema en el país.

En 1981 se diseñó un nuevo plan de estudio, donde se logra una mejor conceptualización del objeto de estudio y de los factores que la condicionan. Este plan se mantuvo vigente (con algunas modificaciones) hasta el año 1993.

El nuevo plan implantado en 1993 se caracterizó por reforzar la formación salubrista y en investigaciones de este especialista, al incrementar los contenidos que le daban salida al perfil de investigaciones. Este plan estuvo vigente hasta el curso 2005-2006, cuando el Ministerio de Salud Pública dispuso una reducción del tiempo de duración de las especialidades médicas a 2 años. Se propuso por tanto a partir de esa fecha un nuevo plan de estudios de dos años de duración para la especialización en Bioestadística. ${ }^{2,3}$

La especialidad cuenta actualmente con 36 años de creada y 219 graduados en 31 graduaciones hasta el curso 2005-2006. Ha transitado, por supuesto, por diferentes etapas de desarrollo a lo largo de estos años, en los cuales han variado las instituciones responsables de dicha figura académica (siempre situadas en La Habana) y por tanto las condiciones materiales en las cuales se ha desarrollado. A pesar de la variabilidad de las instituciones, el claustro ha permanecido bastante constante a lo largo del tiempo.

La evaluación de impacto es particularmente útil en la educación de posgrado ya que este tipo de formación está dirigida fundamentalmente a la capacitación de un profesional destinado a satisfacer de manera inmediata las necesidades concretas del contexto socioeconómico del país atendiendo a las prioridades de su desarrollo científico-técnico, es decir, un profesional del que se espera que su actividad sea realmente útil y trascendente para la sociedad que lo formó.

Medir el impacto educacional resulta complejo ya que el efecto de un proceso de este tipo tiene límites muy difíciles de precisar, pues puede provocar cambios o transformaciones imprescindibles aún para quienes diseñan los programas. Por lo tanto, se hace indispensable delimitar aquellos aspectos sobre los cuales se tratará de medir dicho efecto. ${ }^{4}$

Los diferentes autores que abordan este tema proponen distintas esferas sobre los cuales investigar. Caravia Pubillones alude a la guía propuesta por la Asociación Universitaria I beroamericana de Postgrado, para la autoevaluación del posgrado donde se reconocen ocho variables que pueden ser objeto de evaluación: los alumnos, los egresados, los profesores, el currículo, la administración, el entorno institucional, el impacto social (aporte que el programa hace al desarrollo científico, tecnológico, económico, social y cultural de su entorno), la evaluación con respecto al programa y a su institución. ${ }^{5}$ 
Teniendo en cuenta los conocimientos adquiridos sobre el tema a través de la revisión bibliográfica realizada de estudios anteriores y de las condiciones concretas en que se ha desarrollado la docencia en la especialidad (movilidad institucional y estabilidad del claustro), se decidió entonces evaluar su impacto sobre su claustro y en general sobre la Salud Pública Cubana.

\section{MÉTODOS}

Se realizó una investigación descriptiva, retrospectiva cuyo universo de estudio comprendió a todos los profesores que integran el claustro de la especialidad, así como a 11 expertos en el área de la salud pública pertenecientes a diferentes instituciones: Ministerio de Salud Pública, Instituto Nacional de Higiene, Epidemiología y Microbiología, Hospital "Hermanos Ameijeiras", Dirección Nacional de Estadísticas, Escuela Nacional de Salud Pública, Centro de Cibernética Aplicada a la Medicina, Hospital Militar "Carlos J. Finlay" e Instituto Nacional de Oncología y Radiobiología.

Se construyeron dos modelos de entrevista personal para la obtención de la información: uno dirigido a los profesores integrantes del claustro de la especialidad y el otro a los expertos en el área de la salud pública.

Para construir los instrumentos se identificaron primeramente al claustro y al área de la salud pública como dimensiones sobre la que dicha figura académica pudiera haber repercutido. Esta identificación se realizó a través de revisión bibliográfica y según criterio de las autoras.

Se sometió a consideración de cinco expertos la idoneidad de los modelos propuestos.

El procesamiento de la información proveniente de las entrevistas realizadas se realizó manualmente a través de un análisis de contenido.

\section{RESULTADOS}

\section{I mpacto de la especialidad sobre el claustro que la desarrolla}

Se entrevistaron 22 profesores del claustro de la especialidad. En las entrevistas realizadas hicieron mención de los siguientes aspectos relacionados con la repercusión de su desempeño como profesores de la especialidad en su desarrollo intelectual y laboral.

1. Constituye una de las fuentes más importantes en la formación y desarrollo de los profesores debido a la necesidad de elevación y actualización sistemática en la preparación científica, tecnológica y pedagógica para poder satisfacer las demandas crecientes para la formación de bioestadísticos de mayor calidad.

2. Influencia decisiva en la necesidad de obtener grados científicos superiores. De los 22 profesores encuestados $17(77,3 \%)$ son másteres o se encuentran en proceso de obtención del titulo y $12(54,5 \%)$ son Doctores en Ciencias o se 
encuentran en vías de obtención de dicho grado. Todos han elevado su categoría docente a partir de su inclusión en el claustro.

3. La posibilidad de intercambio con el resto de los profesores sobre temas de la especialidad y la necesidad de asumir paulatinamente nuevas asignaturas contribuye a su desarrollo intelectual y laboral.

4. El ejercicio continuo de la docencia y la labor como tutores y asesores de tesis a través de los años contribuye al perfeccionamiento de su desempeño como investigadores y docentes y por ende a la elevación de la calidad de sus publicaciones científicas y de su intervenciones en proyectos de investigación

5. El hecho mismo de pertenecer a un claustro tan competente y de tan alto nivel científico motiva a los profesores a tratar de alcanzar las categorías científicas y pedagógicas mas elevadas.

\section{I mpacto de la especialidad en el ámbito de la Salud Pública Cubana}

Los entrevistados consideran que el impacto de la especialidad en la Salud Publica Cubana se aprecian en:

1. La calidad de la información estadística que se brinda en todos los niveles del Sistema Nacional de Salud, a la que contribuye la participación de bioestadísticos en el procesamiento de dicha información.

2. Mejoramiento de la calidad de los Análisis de la Situación de Salud a lo que ayuda la información y asesoramiento brindados por los biostadísticos en las diferentes instancias.

3. Aumento de la calidad de las investigaciones relacionado con el aporte de los bioestadísticos en aspectos concernientes a su diseño y ejecución, lo que a su vez propicia el aumento de la cantidad y calidad de las publicaciones científicas realizadas y de la asistencia de profesionales de la salud a eventos científicos.

4. Elevación de la calidad de otros profesionales de la salud como investigadores y docentes (sobre todo en las actividades relacionadas con tutorías y asesorías de tesis) en sus respectivas especialidades a través de la recepción de docencia directa o asesorías de investigaciones por parte de bioestadísticos en sus diferentes puestos de trabajo.

5. Aumento de la integración en equipos de investigación multidisciplinarios debido a que los conocimientos de medicina y estadística que poseen los especialistas en bioestadística posibilita que sean muy útiles para el entendimiento entre médicos y matemáticos.

6. Elevación de la calidad de los eventos científicos realizados en diferentes niveles a lo que colabora el aporte de los bioestadísticos al fortalecimiento del trabajo metodológico en las actividades docentes y de investigación.

7. Creación del Grupo Nacional de Bioestadística.

8. Creación del Comité Académico de la Especialidad.

9. Celebración de la Primera Jornada Nacional de Bioestadística en 1990. 
10. Celebración del I Primer Congreso I beroamericano de Estadísticas Médicas y Segunda Jornada Nacional de Bioestadística en el año 2001.

\section{DISCUSIÓN}

Aunque en el país algunos autores han realizado investigaciones con temas afines a la presente, las esferas exploradas son diferentes, por lo que la confrontación de los ${ }_{8}$ resultados de la presente investigación con los de otros autores se ha hecho dificil. ${ }^{6-}$

Se concluye que la Especialidad de Bioestadística ha tenido impacto sobre el claustro que la desarrolla y que se manifiesta en la elevada preparación científica, tecnológica y pedagógica de sus profesores, así como en su excelente desempeño como investigadores y docentes También ha tenido impacto en la Salud Pública Cubana que se demuestra en la calidad de la información estadística que se brinda en todos los niveles del Sistema Nacional de Salud, en el aumento de la calidad de las investigaciones y eventos científicos realizados en el sector de la salud, en la elevación de la calidad como investigadores y docentes de otros profesionales de la salud, en la creación del Comité Académico y del Grupo Nacional de la Especialidad, así como en la realización de eventos científicos propios de esta disciplina.

\section{REFERENCI AS BI BLI OGRÁFICAS}

1. López Serrano E. Desarrollo histórico de las estadísticas sanitarias en Cuba [serie en Internet]. [citado 10 Ene 2007]. Disponible en:

http://bvs.sld.cu/revistas/his/cua_91/his1491.htm

2. Jiménez R, Astraín ME, Otero Iglesias J, Fernández Barroso L, Castañeda Abascal I, Gran Álvarez M, et. al. Plan de estudio: especialidad de Bioestadística. La Habana. Escuela Nacional Salud Pública, MINSAP; 2002.

3. Jiménez Paneque R, Pría Barros MC, Álvarez Lauserique ME, Coutin MG, Otero I glesias J, Quesada R, et. al. Plan de estudio: especialidad de Bioestadística. La Habana. Escuela Nacional Salud Pública, MINSAP; 2006.

4. Couturejuzón González L. I mpacto institucional y social de la Maestría Informática en Salud. Instituto Superior de Ciencias Médicas de La Habana. 19972002. Rev Cubana Informática Médica [serie en Internet]. 2005 [citado 26 Ago 2008]; 5(3). Disponible en:

http://www. cecam.sld.cu/pages/rcim/revista_9/articulo_9.htm

5. Caravia Pubillones IA. Propuesta de instrumentos para evaluar el impacto de la Maestría de Educación Médica [tesis]. La Habana: Escuela Nacional de Salud Pública; 2005.

6. Diego Olite FM. Evaluación del diplomado a distancia de Salud Ambiental: aspectos pedagógicos e impacto de las funciones sociales de los egresados [tesis]. La Habana: INHEM; 2003. 
7. Cabrera Rodríguez J A. Seguimiento y evaluación de la capacitación y su impacto en el desempeño individual y organizacional [CD-ROM]. La Habana: Universidad Agraria de La Habana "Fructuoso Rodríguez Pérez"; 2003.

8. Álvarez Guevara T. Evaluación de la calidad del diplomado de Salud Escolar y de la competencia y desempeño de los egresados (1997-2001) [tesis]. La Habana: Instituto Superior de Ciencias Médicas de La Habana; 2002.

Recibido: 17 de octubre de 2008.

Aprobado: 22 de octubre de 2008.

Lourdes Couturejuzón González. Escuela Nacional de Salud Pública. Calle Línea esq. I. El Vedado 10400. La Habana, Cuba

E-mail: Icouturejuzon@ensap.sld.cu 